\title{
Improvements in electron-photon-relaxation data for MCNP6
}

\author{
H. Grady Hughes $1{ }^{1, *}$ \\ ${ }^{1}$ Los Alamos National Laboratory, Los Alamos NM 87545 USA
}

\begin{abstract}
After the initial release of the first production version of MCNP6 and its subsequent beta release, the extended electron-photon-relaxation capabilities of the code have continued to be developed. With the availability of newer data, several enhancements and corrections to the methods have been installed into the code. In this paper we discuss these improvements, including the development of three distinct approaches to sampling electron elastic scattering, the correction of an earlier data resolution problem, and a few formal corrections to the earlier ACE data file.
\end{abstract}

\section{Introduction}

The Los Alamos Monte Carlo transport code MCNP6 [1] has recently benefitted from significant work to improve the photon-electron transport and atomic relaxation capabilities of the code. The improvements [2-3] include extension of existing photon data to lower energies than previously supported; addition of new data such as subshell-specific photoelectric cross sections; completion of the incoherent and coherent form factor data to cover all scattering angles at all supported energies; new data for electron interactions including bremsstrahlung, elastic scattering, subshell-specific electroionization, and atomic excitation; a much more detailed representation for atomic relaxation; and an entirely new (to MCNP) approach to electron transport: the single-event method [4]. These improvements were made available with the initial production release of MCNP6 [1] along with an extended electron, photon, and relaxation data file eprdata12 [5] to support the new capabilities.

More recently new data [6] have become available and have now been incorporated into MCNP6, correcting a few issues with the previous work, and adding new sets of cross sections that were not included in eprdata12. The purpose of this paper is to introduce these newer changes and enhancements, which are expected to be released with the next public release of the MCNP6 code system.

\section{Sources of the data}

The database that supports these developments relies on an evolving set of libraries originally released by D. E. Cullen and collaborators [7-9] as separate data for photoatomic interactions (EPDL), atomic relaxation (EADL), and electroatomic interactions (EEDL). An updated version of this release was officially adopted in the ENDF/B VI.8 release of the Evaluated Nuclear Data File [10] and it was this source that was used to produce the ACE (A Compact

\footnotetext{
* Corresponding author: hgh@lanl.gov
}

ENDF) format library eprdata12 [5] for use in the initial production release of MCNP6 (and the subsequent beta release).

The latest improved version of this database was released by Cullen [6] in 2014. This is the EPICS2014 collection, which includes both an ENDF-formatted file and separate EPDL, EADL, and EEDL files. The newest enhancements to the developmental version of MCNP6 are primarily based on improvements and additions drawn from the EPICS2014 data.

\section{Data and code enhancements}

In this section we shall identify and discuss the additions and corrections to the single-event transport method at its current stage of development. There are two changes based on the new data that have potential importance for calculations and two that are primarily formal corrections.

\subsection{Alternate elastic cross sections}

The original eprdata12 presented for each element a tabulation of energy-dependent electron elastic scattering cross sections, together with a collection of secondary angular probability tables at a variety of electron energies. These data actually described the "large-angle" elastic scattering of the electron, specifying the cross section and subsequent deflection angle for electrons experiencing an elastic collision and scattering into a deflection angle in the range $-1<\mu<1-10^{-6}$, i.e. $\theta>\sim 1.4$ milliradians, with $\mu=\cos \theta$. Describing the elastic scattering with only these data is equivalent to making the approximation that scattering within the forward peak can be ignored, treating any scatter within the peak as a delta function in $1-\cos (\theta)$. For many problems, this is a reasonable approximation, and this was all that was available in the initial releases of MCNP6 using eprdata12. 
With the new data of EPICS2014, two more possibilities become available. The first is the provision of a total electron elastic cross section. This is the sum of the large-angle cross section and the cross section for elastic events in which the electron deflection remains within the forward peak. To take advantage of the new data, one can sample for the occurrence of an elastic event using the total rather than the large-angle cross section. Then the ratio of the large-angle to the total cross section can be used in a rejection game to determine the angular sampling method. If the large-angle component is selected, the angular tables are used as in the released versions of MCNP6. If the inpeak component is chosen then the deflection angle is sampled from an analytic probability distribution

$$
\frac{d \sigma}{d \mu}=\frac{A}{(\eta+1-\mu)^{2}}
$$

recommended by the evaluators and given in the ENDF/B VI.8 release. Here $\mu=\cos \theta, A$ is a normalization constant, and $\eta$ is a screening angle used by Berger [11] and by Seltzer [12].

This two-component algorithm, combining both scattering ranges of the elastic interaction, should provide the most physically accurate simulation of the elastic scattering. It also provides the most expensive method in terms of computer time.

The second new possibility comes from the availability in the EPICS2014 data of a transport-corrected cross section for elastic scattering. This construct is often of interest in cases of large, highly forward peaked cross sections such as photon coherent scattering and electron elastic scattering, where its use can greatly reduce the effective cross section (reducing the computational burden) while still preserving the average scattering angle. The use of the transport-corrected cross section is an especially attractive option in highly scattering media, since the large number of elastic events tends to smooth out the details of the angular distribution, making this approach an increasingly good approximation precisely for the problems that need a more efficient computational method.

\subsection{Large-angle near-peak elastic PDF}

One of the issues that created some difficulties with the earlier eprdata12 release was insufficient precision in the tabulation of the angular probability distribution functions for large-angle electron elastic scattering, especially at high energies. Specifically, fewer digits than were needed to resolve the approach to the forward peak were provided in the ENDF/B VI.8 file. For example for $21.25 \mathrm{MeV}$ electrons in iron, the last three points in the tabulation of scattering cosine $v s$ probability density were

$$
\begin{aligned}
& (0.999999,4.60952 \mathrm{E}+05) \\
& (0.999999,6.01183 \mathrm{E}+05) \\
& (0.999999,8.16698 \mathrm{E}+05)
\end{aligned}
$$

This situation clearly prevents the conversion of the angular distribution into a properly integrated, cumulative distribution that can be sampled in standard ways. To overcome this problem, a simple, but rather arbitrary adjustment was made to the data in eprdata12: each sequence of indistinguishable angles was collapsed to a single data point and the average probability density over the sequence was assigned to that point. The resulting cumulative angular distribution then had the expected form, and sampling of the elastic angular deflection could proceed as usual. In practice this was a serviceable expedient, but was hardly satisfactory on theoretical grounds, especially given the eventual availability of data lacking this flaw.

With the release of EPICS2014, this issue is now settled. Both the EEDL and ENDF formatted files of the new library present the tabulations of scattering angle $v s$ probability density with sufficient numerical resolution to eliminate apparently coincident points in the distributions. Thus the preparation of the cumulative distributions and the Monte Carlo sampling for the scattering angle can now be dealt with in the standard way.

\subsection{Average bremsstrahlung energy loss}

It was the intention of the evaluators to provide a tabulation of the average energy of a bremsstrahlung photon as a function of the electron energy at the bremsstrahlung event. In the creation of ENDF/B VI.8, this tabulation was inadvertently reversed to present the average energy remaining to the electron after a bremsstrahlung event at the tabulated electron energy. For consistency with the ENDF release, this definition was retained in eprdata12, although MCNP6 made no actual use of this table. The EPICS2014 release restores the originally intended meaning of the table, so that the tabulated quantity is now the average bremsstrahlung photon energy. This form of the tabulation will also be maintained in the successor to the eprdata12 ACE-format file.

\subsection{Compton Doppler broadening}

The Compton Doppler broadening capability of MCNP allows the simulation of some of the effects of bound electrons on incoherent photon scattering. Data to support this capability have been available in photoatomic libraries including mcplib03 [13], mcplib04 [14] and eprdata12 [5]. Unfortunately, earlier versions of MCNP (MCNP5 and MCNPX) suffered from an inconsistency with the data: the probability that the incoherently scattered photon has interacted with a particular electron shell is presented in the data library as a tabulated probability density function, while the MCNP5 and MCNPX codes treated the table as a cumulative distribution. Thus the older codes produced incorrect results [15] for the distribution of shell interactions. To allow users of earlier versions of MCNP to avoid this bug, two new libraries, mcplib63, and mcplib84 [16] were produced with the probability in question already converted to cumulative form. These libraries can replace mcplib03 and mcplib04, respectively, enabling the older codes to sample Compton Doppler broadening as accurately as the current data can support.

This issue is moot for users of the official releases of MCNP6, since both of those versions automatically detect the form of the shell probability distribution, and convert the table to a cumulative distribution if needed. 
Nevertheless, it seems appropriate to keep the eprdata library consistent with the newest of the traditional photoatomic libraries. Therefore the successor to eprdata12 will present the shell probabilities for Compton Doppler broadening as a cumulative distribution.

\section{Ongoing developments}

We are currently undertaking a broad review of benchmark calculations suitable for verification and validation of the electron-photon transport algorithms and data in MCNP6. This study will cover both the condensed-history and the single-event methods for electron transport, as well as the data and sampling methods for atomic relaxation. It is especially important that the newer data for electron elastic scattering be verified and validated, and that the practical computational aspects of the new methods be explored.

We are also conducting reviews of specific aspects of the transport, including the possibility of improvements in the details of the condensed-history algorithms, especially by increasing the use of the new eprdata libraries in several areas of condensed-history, where they have not previously been used.

\section{Summary}

We have provided a brief discussion of four recent enhancements and corrections to the electron, photon, and atomic relaxation data of the eprdata series of data libraries included with the MCNP6 distribution. These data and associated code improvements are undergoing verification and validation testing, and are projected to be a part of the next official release of the MCNP6 code system. A future extended version of this paper will present specific examples of the new capabilities and the results of practical calculations.

\section{References}

1. T. Goorley, M. James, T. Booth, F. Brown, J. Bull, L. J. Cox, J. Durkee, J. Elson, M. Fensin, R. A. Forster, J. Hendricks, H. G. Hughes, R. Johns, B. Kiedrowski, R. Martz, S. Mashnik, G. McKinney, D. Pelowitz, R. Prael, J. Sweezy, L. Waters, T. Wilcox, T. Zukaitis, Nuc. Tech. 180 298-315 (2012).

2. G. Hughes, Prog. Nuc. Sci. and Tech. 4 454-458 (2014)

3. H. G. Hughes, $S N A+M C 2013,03105$ (2014)

4. H. G. Hughes, Los Alamos National Laboratory report LA-UR-12-21068, Rev. 3 (2013)

5. H. G. Hughes, Los Alamos National Laboratory report LA-UR-13-27377, Rev. 1 (2015)

6. D. E. Cullen, IAEA-NDS-218, IAEA Nuc. Data Section (2014)

7. D. E. Cullen, M. H. Chen, J. H. Hubbell, S. T. Perkins, E. F. Plechaty, J. A. Rathkopf, J. H. Scofield, Lawrence Livermore National Laboratory report UCRL-50400 6 Parts A-B, Rev. 4 (1989)
8. S. T. Perkins, D. E. Cullen, M. H. Chen, J. H. Hubbell, J. Rathkopf, J. Scofield, Lawrence Livermore National Laboratory report UCRL-50400 30 (1991)

9. S. T. Perkins, D. E. Cullen, and S. M. Seltzer, Lawrence Livermore National Laboratory report UCRL-50400 31 (1991)

10. Cross Section Evaluation Working Group, Brookhaven National Laboratory report BNL-NCS17541, 8th Ed. (2000)

11. M. J. Berger, R. Wang, in Monte Carlo Transport of Electrons and Photons Ch. 2 (Plenum Press, New York, 1988)

12. S. M. Seltzer, Ibid. Ch. 7

13. M. C. White, Los Alamos National Laboratory report LA-UR-03-0787 (2003)

14. M. C. White, Los Alamos National Laboratory report LA-UR-03-1019 (2003)

15. B. C. Kiedrowski, F. B. Brown, M. C. White, D. K. Parsons, Los Alamos National Laboratory report LAUR-12-00121 (2012)

16. M. C. White, Los Alamos National Laboratory report LA-UR-12-00018 (2012) 